\title{
Uric acid and incident dementia over 12 years of follow-up: a population-based cohort study
}

\author{
Augustin Latourte, 1,2,3 Aicha Soumaré, ${ }^{4}$ Thomas Bardin, ${ }^{1,2,3}$ Fernando Perez-Ruiz, ${ }^{5}$ \\ Stéphanie Debette, ${ }^{4,6,7,8}$ Pascal Richette ${ }^{1,2,3}$
}

- Additional material is

published online only. To view please visit the journal online (http://dx.doi.org/10.1136/ annrheumdis-2016-210767).

${ }^{1}$ Université Paris Diderot, UFR médicale, Paris, France

${ }^{2}$ Hôpital Lariboisière, Service de Rhumatologie, Paris, Cedex, France

${ }^{3}$ INSERM 1132, Université ParisDiderot, Hôpital Lariboisière, Paris, France

${ }^{4}$ Inserm Centre Bordeaux Population Health (U1219),

Bordeaux, France

${ }^{5}$ Division of Rheumatology, Hospital Universitario Cruces, Biocruces Health Research Institute, and Basque Country University, Biscay, Spain ${ }^{6}$ University of Bordeaux, Bordeaux, France ${ }^{7}$ Department of Neurology, Memory Clinic, Bordeaux, France

${ }^{8}$ Department of Neurology, Framingham Heart Study, Boston University School of Medicine, Boston, Massachusetts, USA

\section{Correspondence to} Pascal Richette, Fédération de Rhumatologie, Centre Viggo Petersen, Hôpital Lariboisière, 2 rue Ambroise Paré, 75010 Paris, France; pascal.richette@aphp.fr

SD and PR contributed equally, $\mathrm{AL}$ and $\mathrm{AS}$ contributed equally.

Received 3 November 2016 Revised 23 June 2017 Accepted 26 June 2017 Published Online First 28 July 2017

\section{Linked}

- http://dx.doi.org/10.1136/ annrheumdis-2017-211975

-

Check for updates

To cite: Latourte $\mathrm{A}$,

Soumaré A, Bardin T

et al. Ann Rheum Dis

2018;77:328-335.

\section{ABSTRACT}

Objectives In patients with gout, maintaining too low serum uric acid (SUA) level with urate-lowering therapy is a concern because uric acid is thought to be neuroprotective. However, the relation between SUA and dementia remains debated. This study aimed to investigate the impact of SUA level on the incidence of dementia.

Methods We assessed the longitudinal association between SUA level and incident dementia (Diagnostic and Statistical Manual of Mental Disorders Version IV (DSM-IV) criteria) in a large cohort of healthy older people from the community (Three-City Dijon cohort). Additionally, we investigated the relation between SUA level and MRI markers of brain ageing (white matter hyperintensity volume (WMHV), lacunes and hippocampal volume).

Results The study sample comprised 1598 people (mean (SD) age 72.4(4.1) years, 38.3\% male). During the 13,357 person-years of follow-up (median duration: 10.1 years), dementia developed in 110 participants (crude incidence rate: 8.2/1000 person-years). After multiple adjustments, the multivariate HR with the highest ( $\geq 75$ th percentile) versus lowest SUA level was $1.79(95 \% \mathrm{Cl}$ 1.17 to $2.73 ; p=0.007)$. The association was stronger with vascular or mixed dementia (HR=3.66 $(95 \% \mathrm{Cl} 1.29$ to 10.41$), p=0.015)$ than Alzheimer's disease ( $H R=1.55$ $(95 \% \mathrm{Cl} 0.92$ to 2.61$), p=0.10)$. There was a nonsignificant trend towards an association between high SUA level and extensive WMHV ( $p=0.10)$, a biomarker of small vessel disease, but not hippocampal volume $(p=0.94)$ or lacunes $(p=0.86)$. The association between SUA level and vascular or mixed dementia might be affected by interim strokes,

Conclusions Risk of dementia, especially vascular or mixed dementia, may be increased with high SUA levels in elderly people.

\section{INTRODUCTION}

Hyperuricemia is a prerequisite for gout and might be a risk factor for cardiovascular and kidney diseases. ${ }^{1-3}$ For treating gout, the European League Against Rheumatism and American College of Rheumatology recommend lowering serum uric acid (SUA) level below $360 \mu \mathrm{mol} / \mathrm{L}$ and below 300 $\mu \mathrm{mol} / \mathrm{L}$ in some cases. ${ }^{45}$ However, maintaining too low SUA levels is a concern because uric acid (UA) is thought to be neuroprotective. ${ }^{6} \mathrm{UA}$ might have important antioxidant properties, ${ }^{7}$ and some studies have reported increased risk of several neurodegenerative diseases such as Parkinson's disease ${ }^{8}$ or amyotrophic lateral sclerosis with a low SUA level. ${ }^{9}$
The association of SUA level with cognitive impairment and dementia has been explored, but results are conflicting. ${ }^{6}$ The risk of Alzheimer's disease (AD) was found inversely associated with SUA level and gout, ${ }^{10-12}$ with less convincing associations with other subtypes of dementia, such as vascular dementia. ${ }^{6}{ }^{12}$ Furthermore, given the putative adverse effect of UA on cardiovascular outcomes, UA may contribute to cognitive impairment and dementia risk via increased risk of cerebrovascular disease. ${ }^{13}$ Most studies that investigated the link between SUA level and dementia were cross-sectional, with risk of bias (review in refs 6 and 10), and longitudinal studies are scarce. Thus, more longitudinal population-based cohort studies are needed to better unravel the complex link between UA and cognitive impairment.

Brain changes occur silently over a long time before clinical features of dementia become evident. ${ }^{14}$ Therefore, an assessment of the impact of SUA level on incident dementia needs to also investigate preclinical biomarkers, with an extended follow-up. Brain MRI markers such as hippocampal atrophy or white matter hyperintensities (WMHs) are known strong predictors of further cognitive impairment and are thus of particular interest for exploring the link between SUA level and neurodegenerative disorders. ${ }^{14-16}$ Here we investigated the risk of incident dementia and brain MRI features by SUA level in a large cohort of older adults.

\section{METHODS}

\section{Study population}

The Three-City Dijon Study (3C-Dijon) is a French population-based cohort study of 4931 non-institutionalised people aged $\geq 65$ years old who were randomly selected from the city electoral rolls. ${ }^{17}$ Baseline examination (January 1999 to March 2001) consisted of a face-to-face interview (for collecting sociodemographic and lifestyle characteristics and medical history), fasting blood sampling and physical and cognitive examination. In addition, between June 1999 and September 2000 , participants who were aged $<80$ years old $(n=2763)$ were asked to undergo a cerebral MRI; 2285 subjects agreed (83\%) and 1924 scans were performed at baseline.

Over a 12 -year period, six follow-up visits took place at a 2-year interval. The third follow-up examination consisted of a self-reporting questionnaire or a phone interview for participants who had refused to or could not complete the questionnaire. Written informed consent was obtained from all 
participants, and the study protocol was approved by the ethics committee of the University Hospital of Kremlin-Bicêtre.

To be eligible for the present study, participants had to have a baseline assessment of SUA level, no clinical diagnosis for dementia at baseline, a Mini-Mental State Examination (MMSE) score of $>24$ to exclude potential undiagnosed dementia and at least one follow-up visit. We excluded participants receiving urate-lowering treatments (ULT) because they were likely to have gout $(n=61$; their baseline characteristics are described in online supplementary table 1), a diagnosis that might interfere with the diagnosis of dementia according to recent studies. ${ }^{11}{ }^{12}$ In all, 1598 participants met these criteria and represented the study sample for dementia analyses. After excluding participants with brain tumours $(\mathrm{n}=8)$, our baseline sample for MRI markers consisted of those with baseline SUA measurements and valid MRI data on WMH volume (WMHV; $\mathrm{n}=1525)$, MRI-defined brain infarcts $(\mathrm{n}=1588)$ and hippocampal volume $(\mathrm{n}=1393)$.

\section{Screening and diagnosis of dementia}

Diagnosis of dementia was based on a classical three-step procedure. At each follow-up examination (2, 4, 8, 10 and 12 years after enrolment), trained psychologists screened participants for dementia by using the MMSE and the Isaacs Set Test. Participants were further examined by a physician who performed additional neuropsychological testing and assessed the degree of impairment. Finally, an independent committee of neurologists reviewed all potential prevalent and incident cases of dementia to reach consensus on the diagnosis and aetiology, in accordance with DSM-IV criteria. For AD, the dementia classification was based on the criteria of the National Institute of Neurological and Communicative Disorders and Stroke-Alzheimer's Disease and Related Disorders Association (NINCDS-ADRDA) and for vascular dementia, on the criteria of the National Institute of Neurological Disorders and the Stroke-Association Internationale pour la Recherche et l'Enseignement en Neurosciences (NINDS-AIREN). ${ }^{18} 19$ In our study, mixed dementia (AD with vascular lesions) and pure vascular dementia were combined to form a category of dementia with a vascular component.

\section{Brain MRI and variables definitions}

MRI acquisition was performed on 1.5-Tesla Magnetom scanner (Siemens, Erlangen). One experienced and independent radiologist blinded to clinical data performed the MRI readings for brain infarcts and lacunes detection. Further details regarding MRI acquisition and assessment of other brain lesions and volumes are described in online supplementary material.

\section{Measurement of urate levels and other measurements (see online supplementary material)}

\section{Statistical analyses}

SUA levels are consistently lower in females than in males over time, even after menopause. ${ }^{20}$ This was the case in the 3C-Dijon study (age-adjusted mean SUA (SE): 305.1 (2.7) in males vs $254.2(2.1)$ in females, $\mathrm{p}<0.0001)$. In order to avoid any potential confounder related to gender, we generated SUA quantiles using gender-dependent thresholds in our main analysis: 75 th percentile $=345 \mu \mathrm{mol} / \mathrm{L}$ for men, $292 \mu \mathrm{mol} / \mathrm{L}$ for women; quartile 1 (Q1): $<260 \mu \mathrm{mol} / \mathrm{L}$ for $\mathrm{men},<209 \mu \mathrm{mol} / \mathrm{L}$ for women; quartile 2 (Q2): 260-299 for men, 209-247 for women; quartile 3 (Q3): 299-345 for men, 247-292 for women; quartile 4
Table 1 Baseline characteristics of the study sample and association with baseline SUA level

\begin{tabular}{|c|c|c|c|c|}
\hline & \multirow{2}{*}{$\begin{array}{l}\begin{array}{l}\text { Total } \\
\text { sample, }\end{array} \\
\mathrm{n}=1598\end{array}$} & \multicolumn{2}{|c|}{ Baseline high SUA level ${ }^{*}$} & \multirow[b]{2}{*}{ pt } \\
\hline & & No $(n=1192)$ & Yes $(n=406)$ & \\
\hline Age, years, mean (SD) & $72.4(4.1)$ & $72.2(4.1)$ & $72.9(4.1)$ & 0.002 \\
\hline Male & $612(38.3)$ & $455(38.2)$ & $157(38.7)$ & 0.86 \\
\hline High education levelł & $654(40.9)$ & $501(42.0)$ & $153(37.7)$ & 0.12 \\
\hline $\begin{array}{l}\text { Current drinker } \\
\text { (>2 drinks/week) }\end{array}$ & $332(22.6)$ & $231(21.1)$ & $101(27.2)$ & 0.07 \\
\hline Current smoking & $92(5.8)$ & $67(5.6)$ & $25(6.2)$ & 0.008 \\
\hline $\mathrm{BMI}, \mathrm{kg} / \mathrm{m}^{2}$, mean (SD) & $25.4(3.8)$ & $24.8(3.7)$ & $27.1(3.7)$ & $<0.0001$ \\
\hline $\begin{array}{l}\text { Glomerular filtration rate, } \\
\mathrm{mL} / \mathrm{min} / 1.73 \mathrm{~m}^{2} \text {, mean } \\
\text { (SD) }\end{array}$ & $75.1(13.7)$ & $76.8(13.3)$ & $70.2(13.7)$ & $<0.0001$ \\
\hline Hypertension & $1209(75.7)$ & $861(72.2)$ & $348(85.7)$ & $<0.0001$ \\
\hline Diabetes mellitus & $121(7.6)$ & $87(7.3)$ & $34(8.4)$ & 0.47 \\
\hline $\begin{array}{l}\text { History of cardiovascular } \\
\text { disease }\end{array}$ & $91(5.7)$ & $57(4.8)$ & $34(8.4)$ & 0.007 \\
\hline NSAIDs use & $239(15.1)$ & $178(15.1)$ & $61(15.1)$ & 0.99 \\
\hline $\begin{array}{l}\text { Concomitant use of } \\
\text { aspirin or diuretics }\end{array}$ & $424(26.6)$ & $270(22.7)$ & $154(38.0)$ & $<0.0001$ \\
\hline APOE $\varepsilon 4$ carrier & $347(21.7)$ & $269(22.6)$ & $78(19.2)$ & 0.16 \\
\hline $\begin{array}{l}\text { LDL-c level, mmol/L, mean } \\
\text { (SD) }\end{array}$ & $3.58(0.81)$ & $3.59(0.81)$ & $3.55(0.83)$ & 0.38 \\
\hline $\begin{array}{l}\text { HDL-c level, mmol/L, } \\
\text { mean (SD) }\end{array}$ & $1.65(0.40)$ & $1.68(0.40)$ & $1.55(0.40)$ & $<0.0001$ \\
\hline $\begin{array}{l}\text { Triglycerides level, } \\
\mathrm{mmol} / \mathrm{L} \text {, mean (SD) }\end{array}$ & $1.20(0.56)$ & $1.13(0.51)$ & $1.40(0.62)$ & $<0.0001$ \\
\hline High CRP level§ & $537(33.6)$ & $375(31.5)$ & $162(39.9)$ & 0.005 \\
\hline High IL-6 levelף & $411(25.7)$ & $278(23.3)$ & $133(32.8)$ & $<0.0001$ \\
\hline $\begin{array}{l}\text { SUA level, } \mu \mathrm{mol} / \mathrm{L} \text {, mean } \\
\text { (SD) }\end{array}$ & $273.7(70.4)$ & $244.2(48.3)$ & $360.3(51.1)$ & $<0.0001$ \\
\hline
\end{tabular}

${ }^{*}$ SUA level $\geq 75$ th percentile of distribution, gender dependent $(345 \mu \mathrm{mol} / \mathrm{L}$ for men; $292 \mu \mathrm{mol} / \mathrm{L}$ for women).

†Analysis of covariance for continuous variables; $\chi^{2}$ test for categorical variables. ¥High school or university diploma.

$\S C R P$ level $\geq 66$ th percentile of distribution $(\geq 2.49 \mathrm{mg} / \mathrm{L})$.

IIIL-6 level $\geq$ median of distribution ( $\geq 4.0 \mathrm{pg} / \mathrm{mL}$ ).

APOE, apolipoprotein E; BMI, body mass index; CRP, C reactive protein; HDL-c, high-density lipoprotein cholesterol; IL, interleukin; LCL-c, low-density lipoprotein cholesterol; NSAIDs, non-steroidal anti-inflammatory drugs; SUA, serum uric acid.

Values are number (percentage) unless stated otherwise.

(Q4): $\geq 345$ for men, $\geq 292$ for women. We described the characteristics of the cohort by SUA level at baseline by using analysis of covariance for continuous variables and $\chi^{2}$ test for categorical variables.

We assessed the risk of our primary outcome, dementia (all-cause, $\mathrm{AD}$, vascular or mixed) according to SUA levels by using Cox proportional hazard models with age as a time scale and calculated HRs and their 95\% CIs. Because of difficulties in defining the date of onset of the dementia process, which is generally insidious, we defined the age of diagnosis of dementia as the age at the midpoint of the interval between the date of diagnosis and the date of the previous visit without dementia. Data were censored at age of diagnosis of dementia (for dementia cases) or last follow-up visit (for non-dementia cases).

For further details regarding statistical analyses see online supplementary material. All analyses involved use of SAS V.9.3 (SAS Institute, Cary, North Carolina, USA), and a two-tailed $p$ value $\leq 0.05$ was considered statistically significant. 
Clinical and epidemiological research

Table 2 Association of baseline SUA level and incident all-cause dementia and by type

\begin{tabular}{|c|c|c|c|c|c|c|c|c|c|}
\hline & $\%$ & Model $1 *$ & & Model $2 \dagger$ & & Model $3 \ddagger$ & & Model $4 \S$ & \\
\hline UA level $(\mu \mathrm{mol} / \mathrm{L})$ & ( $\mathrm{N}$ event) & HR $(95 \% \mathrm{Cl})$ & $\mathrm{p}$ & HR $(95 \% \mathrm{Cl})$ & $\mathrm{p}$ & HR $(95 \% \mathrm{Cl})$ & $\mathrm{p}$ & HR $(95 \% \mathrm{Cl})$ & $\mathrm{p}$ \\
\hline \multicolumn{10}{|c|}{ All-cause dementia $(n=110)$} \\
\hline$<75$ th percentile & $5.7(68)$ & 1.00 (ref.) & & 1.00 (ref.) & & 1.00 (ref.) & & 1.00 (ref.) & \\
\hline$\geq 75$ th percentile & $10.3(42)$ & $1.67(1.13 ; 2.47)$ & 0.009 & $1.81(1.18 ; 2.75)$ & 0.006 & $1.79(1.17 ; 2.73)$ & 0.007 & $1.86(1.21 ; 2.86)$ & 0.004 \\
\hline Q1 & $4.8(19)$ & 1.00 (ref.) & & 1.00 (ref.) & & 1.00 (ref.) & & 1.00 (ref.) & \\
\hline Q2 & $6.0(24)$ & $1.12(0.61 ; 2.04)$ & 0.72 & $1.18(0.64 ; 2.19)$ & 0.60 & $1.20(0.65 ; 2.22)$ & 0.57 & $1.27(0.68 ; 2.37)$ & 0.45 \\
\hline Q3 & $6.3(25)$ & $1.30(0.72 ; 2.36)$ & 0.39 & $1.42(0.77 ; 2.62)$ & 0.27 & $1.46(0.79 ; 2.71)$ & 0.23 & $1.62(0.87 ; 3.03)$ & 0.13 \\
\hline Q4 & $10.3(42)$ & $1.90(1.10 ; 3.29)$ & 0.021 & $2.20(1.21 ; 3.98)$ & 0.009 & $2.21(1.22 ; 4.00)$ & 0.009 & $2.43(1.34 ; 4.42)$ & 0.004 \\
\hline$p$ for linear trend & & & 0.008 & & 0.004 & & 0.004 & & 0.001 \\
\hline \multicolumn{10}{|c|}{ Alzheimer's disease $(n=76)$} \\
\hline$<75$ th percentile & $4.2(49)$ & 1.00 (ref.) & & 1.00 (ref.) & & 1.00 (ref.) & & 1.00 (ref.) & \\
\hline$\geq 75$ th percentile & $6.9(27)$ & $1.48(0.92 ; 2.39)$ & 0.11 & $1.55(0.92 ; 2.59)$ & 0.10 & $1.55(0.92 ; 2.61)$ & 0.10 & $1.61(0.96 ; 2.72)$ & 0.07 \\
\hline Q1 & $3.8(15)$ & 1.00 (ref.) & & 1.00 (ref.) & & 1.00 (ref.) & & 1.00 (ref.) & \\
\hline Q2 & $3.8(15)$ & $0.88(0.43 ; 1.81)$ & 0.74 & $0.90(0.43 ; 1.87)$ & 0.77 & $0.92(0.44 ; 1.92)$ & 0.82 & $0.99(0.47 ; 2.10)$ & 0.99 \\
\hline Q3 & $4.9(19)$ & $1.25(0.63 ; 2.45)$ & 0.52 & $1.31(0.65 ; 2.64)$ & 0.45 & $1.35(0.67 ; 2.73)$ & 0.41 & $1.52(0.74 ; 3.11)$ & 0.26 \\
\hline Q4 & $6.9(27)$ & $1.54(0.81 ; 2.91)$ & 0.19 & $1.66(0.83 ; 3.33)$ & 0.15 & $1.70(0.84 ; 3.42)$ & 0.14 & $1.89(0.94 ; 3.83)$ & 0.08 \\
\hline$p$ for linear trend & & & 0.09 & & 0.07 & & 0.07 & & 0.06 \\
\hline \multicolumn{10}{|c|}{ Vascular or mixed dementia $(n=20)$} \\
\hline$<75$ th percentile & $0.8(9)$ & 1.00 (ref.) & & 1.00 (ref.) & & 1.00 (ref.) & & 1.00 (ref.) & \\
\hline$\geq 75$ th percentile & $2.9(11)$ & $3.11(1.29 ; 7.53)$ & 0.012 & $3.74(1.35 ; 10.36)$ & 0.011 & $3.66(1.29 ; 10.41)$ & 0.015 & $3.59(1.26 ; 10.20)$ & 0.017 \\
\hline Q1 & $0.5(2)$ & 1.00 (ref.) & & 1.00 (ref.) & & 1.00 (ref.) & & 1.00 (ref.) & \\
\hline Q2 & $1.3(5)$ & $1.98(0.38 ; 10.22)$ & 0.42 & $2.72(0.52 ; 14.34)$ & 0.94 & $2.88(0.54 ; 15.40)$ & 0.22 & $2.97(0.56 ; 15.89)$ & 0.20 \\
\hline Q3 & $0.5(2)$ & $0.97(0.14 ; 6.87)$ & 0.97 & $1.34(0.18 ; 9.96)$ & 0.78 & $1.41(0.19 ; 10.55)$ & 0.74 & $1.42(0.19 ; 10.82)$ & 0.74 \\
\hline Q4 & $2.9(11)$ & $4.25(0.94 ; 19.24)$ & 0.06 & $6.38(1.23 ; 33.26)$ & 0.028 & $6.47(1.21 ; 34.43)$ & 0.029 & $6.41(1.20 ; 34.29)$ & 0.03 \\
\hline$p$ for linear trend & & & 0.025 & & 0.017 & & 0.021 & & 0.022 \\
\hline
\end{tabular}

${ }^{*}$ Model 1: adjusted for education level, gender.

†Model 2: model 1 + BMI, tobacco and alcohol consumption, cholesterol (LDL and HDL), triglycerides, diabetes mellitus, hypertension, interaction between hypertension and time (age at last follow-up or dementia occurrence), history of cardiovascular disease, glomerular filtration rate and APOE-ع4.

¥Model 3: model 2 + NSAIDs, aspirin or diuretics.

§Model 4: model 3 + CRP and IL-6 levels, and interaction between IL-6 and time (age at last follow-up or dementia occurrence).

APOE, apolipoprotein E; BMI, body mass index; CRP, C reactive protein; HDL, high-density lipoprotein; IL, interleukin; LDL, low-density lipoprotein; NSAIDs, non-steroidal antiinflammatory drugs; SUA, serum uric acid.

Q1: quartile 1 (lowest quartile); Q2: quartile 2; Q3: quartile 3; Q4: quartile 4 (highest quartile). Cutoffs gender-specific for SUA: 75th percentile=345 for men, 292 for women;

Q1: <260 in men, <209 for women; Q2: 260-299 for men, 209-247 for women; Q3: 299-345 for men, 247-292 for women; Q4: $\geq 345$ for men, $\geq 292$ for women.

\section{RESULTS}

\section{Study sample}

The study population involved 1598 people (mean (SD) age 72.4 (4.1) years, males 38.3\%) (table 1). The mean SUA level at baseline was $273.7 \pm 70.4 \mu \mathrm{mol} / \mathrm{L}$. High baseline SUA level was associated with older age, history of cardiovascular disease and usual cardiovascular risk factors (currently smoking, high body mass index (BMI), hypertension, low high-density lipoprotein cholesterol level and high triglycerides levels), low glomerular filtration rate, aspirin or diuretics use, high levels of $\mathrm{C}$ reactive protein and interleukin (IL)-6 (table 1).

During the 13357 person-years of follow-up (median follow-up: 10.1 years), dementia developed in 110 participants (crude incidence rate: 8.2/1000 person-years): 76 cases (69.1\%) classified as $\mathrm{AD}$ and $20(18.2 \%)$ vascular or mixed dementia (table 2). Incident dementia was associated with older age (mean $74.7(4.1)$ vs $72.2(4.1)$ years, $\mathrm{p}<0.0001)$ and apolipoprotein E (APOE)- $\varepsilon 4$ carriage $(37.3 \%$ vs $20.6 \%, p<0.0001)$ (see online supplementary table 2 ).

\section{SUA levels and incident dementia (primary outcome)}

Risk of dementia was increased with the highest quartile of SUA level $(\geq 345 \mu \mathrm{mol} / \mathrm{L}$ for men, $\geq 292 \mu \mathrm{mol} / \mathrm{L}$ for women) as compared with the lowest quartile $(<260 \mu \mathrm{mol} / \mathrm{L}$ for men, $<209$ $\mu \mathrm{mol} / \mathrm{L}$ for women) $(\mathrm{HR}=1.90$ (95\% CI 1.10 to 3.29$), \mathrm{p}=0.008$ for trend, model 1) (table 2). The association persisted after adjustment for traditional cardiovascular risk factors $(\mathrm{HR}=2.2$ (1.21 to $3.98), p=0.004$ for trend, model 2) and with further adjustment for drugs affecting SUA level or NSAIDs in model $3(\mathrm{HR}=2.21$ (1.22 to 4.00$), p=0.004$ for trend) or inflammatory markers in model 4 ( $\mathrm{HR}=2.43$ (1.34 to 4.42), $\mathrm{p}=0.001$ for trend).

The association of dementia and SUA level was stronger with vascular or mixed dementia ( $\mathrm{HR}=6.41$ (1.20 to 34.29), $\mathrm{p}$ for trend $=0.022$; model 4$)$ than $\mathrm{AD}(\mathrm{HR}=1.89(0.94$ to 3.83$)$, $\mathrm{p}$ for trend $=0.06$; model 4 ). The association between SUA levels and all-cause dementia was also maintained when we used gender-unified quartiles for SUA levels (see online supplementary table 3 ).

\section{SUA levels and brain MRI markers (secondary outcomes)}

We did not find any association between SUA levels and MRI markers of cerebrovascular disease (table 3), or hippocampal volume (model 4, $\beta$ (SE) $=-0.003(0.040), p=0.94$ for high SUA level ( $\geq 75$ th percentile)).

\section{Sensitivity analyses}

When the association between SUA and dementia was examined in subjects not taking NSAIDs, the association with AD was 
Table 3 Association of baseline SUA level and baseline MRI brain markers of cerebrovascular disease

\begin{tabular}{|c|c|c|c|c|c|c|c|c|c|c|}
\hline $\begin{array}{l}\text { Extensive } \\
\text { WMHV }\end{array}$ & $\mathrm{SUA}(\mu \mathrm{mol} / \mathrm{L})$ & $\%(\mathrm{~N})$ & \multicolumn{2}{|l|}{ Model $1^{*}$} & \multicolumn{2}{|l|}{ Model $2 \dagger$} & \multicolumn{2}{|l|}{ Model 3‡ } & \multicolumn{2}{|l|}{ Model $4 \S$} \\
\hline & $<75$ th percentile & $22.8(260)$ & 1.00 (ref.) & & 1.00 (ref.) & & 1.00 (ref.) & & 1.00 (ref.) & \\
\hline & $\geq 75$ th percentile & $31.3(120)$ & $1.53(1.18 ; 1.98)$ & 0.001 & $1.29(0.97 ; 1.71)$ & 0.08 & $1.27(0.95 ; 1.69)$ & 0.10 & $1.23(0.92 ; \mathrm{v} 1.64)$ & 0.16 \\
\hline & Q1 & $21.7(83)$ & 1.00 (ref.) & & 1.00 (ref.) & & 1.00 (ref.) & & 1.00 (ref.) & \\
\hline & Q2 & $21.4(82)$ & $0.98(0.69 ; 1.38)$ & 0.89 & $0.95(0.67 ; 1.36)$ & 0.79 & $0.96(0.67 ; 1.37)$ & 0.80 & $0.96(0.67 ; 1.37)$ & 0.81 \\
\hline & $p$ for linear trend & & & 0.0008 & & 0.07 & & 0.10 & & 0.15 \\
\hline \multirow[t]{4}{*}{ Brain infarcts } & SUA ( $\mu \mathrm{mol} / \mathrm{L})$ & $\%(\mathrm{~N})$ & OR $(95 \% \mathrm{Cl})$ & $p$ & OR $(95 \% \mathrm{Cl})$ & $p$ & OR $(95 \% \mathrm{Cl})$ & $\mathrm{p}$ & OR $(95 \% \mathrm{Cl})$ & $p$ \\
\hline & $<75$ th percentile & $9.1(108)$ & 1.00 (ref.) & & 1.00 (ref.) & & 1.00 (ref.) & & 1.00 (ref.) & \\
\hline & $\geq 75$ th percentile & $11.6(47)$ & $1.22(0.85 ; 1.77)$ & 0.28 & $0.94(0.62 ; 1.42)$ & 0.77 & $0.92(0.60 ; 1.39)$ & 0.68 & $0.90(0.59 ; 1.36)$ & 0.61 \\
\hline & Q1 & $7.4(29)$ & 1.00 (ref.) & & 1.00 (ref.) & & 1.00 (ref.) & & 1.00 (ref.) & \\
\hline \multirow[t]{8}{*}{ Lacunes } & $\mathrm{SUA}(\mu \mathrm{mol} / \mathrm{L})$ & $\%(\mathrm{~N})$ & OR $(95 \% \mathrm{Cl})$ & $p$ & OR $(95 \% \mathrm{Cl})$ & $\mathrm{p}$ & OR $(95 \% \mathrm{Cl})$ & $\mathrm{p}$ & OR $(95 \% \mathrm{Cl})$ & $\mathrm{p}$ \\
\hline & $<75$ th percentile & $7.0(81)$ & 1.00 (ref.) & & 1.00 (ref.) & & 1.00 (ref.) & & 1.00 (ref.) & \\
\hline & $\geq 75$ th percentile & $9.4(37)$ & $1.30(0.86 ; 1.97)$ & 0.22 & $0.99(0.63 ; 1.57)$ & 0.98 & $0.96(0.60 ; 1.53)$ & 0.86 & $0.94(0.59 ; 1.50)$ & 0.79 \\
\hline & Q1 & $5.2(20)$ & 1.00 (ref.) & & 1.00 (ref.) & & 1.00 (ref.) & & 1.00 (ref.) & \\
\hline & Q2 & $6.2(24)$ & $1.20(0.65 ; 2.23)$ & 0.56 & $1.16(0.61 ; 2.18)$ & 0.65 & $1.17(0.62 ; 2.21)$ & 0.63 & $1.18(0.62 ; 2.24)$ & 0.61 \\
\hline & Q3 & $9.6(37)$ & $1.99(1.12 ; 3.52)$ & 0.018 & $1.80(0.99 ; 3.25)$ & 0.05 & $1.79(0.98 ; 3.24)$ & 0.06 & $1.80(0.99 ; 3.29)$ & 0.05 \\
\hline & Q4 & $9.4(37)$ & $1.80(1.01 ; 3.18)$ & 0.044 & $1.35(0.72 ; 2.54)$ & 0.35 & $1.31(0.69 ; 2.48)$ & 0.41 & $1.29(0.68 ; 2.45)$ & 0.43 \\
\hline & $p$ for linear trend & & & 0.023 & & 0.29 & & 0.36 & & 0.40 \\
\hline
\end{tabular}

${ }^{*}$ Model 1: adjusted for age, gender.

tModel 2: model 1 + BMI, tobacco and alcohol consumption, cholesterol (LDL and HDL), triglycerides, diabetes mellitus, hypertension, history of cardiovascular disease, glomerular filtration rate.

¥Model 3: model $2+$ NSAIDs, aspirin or diuretics.

§Model 4: model $3+$ CRP and IL-6 levels.

BMI, body mass index; CRP, C reactive protein; HDL, high-density lipoprotein; IL, interleukin; LDL, low-density lipoprotein; NSAIDs, non-steroidal anti-inflammatory drugs; SUA, serum uric acid; WMHV, white matter hyperintensity volume.

Q1: quartile 1 (lowest quartile); Q2: quartile 2; Q3: quartile 3; Q4: quartile 4 (highest quartile). Cutoffs gender-specific for SUA: 75 th percentile=345 for men, 292 for women;

Q1: <260 for men, <209 for women; Q2: 260-299 for men, 209-247 for women; Q3: 299-345 for men, 247-292 for women; Q4: $\geq 345$ for men, $\geq 292$ for women. Extensive

WMHV is defined as the age-specific top quartile ( $\geq 75$ th percentile) of WMHV over white mask volume (thresholds are calculated in three-age strata: $<70$, (70-75)

and $\geq 75$ years). Logistic regression models were used to assess the probability of having brain infarcts, lacunes or extensive WMHV at baseline by baseline SUA level.

strengthened and the one with vascular or mixed dementia lost its significance (table 4). When the usual definition of hyperuricemia was used as cutoff $(\geq 360 \mu \mathrm{mol} / \mathrm{L}$ in males, $\geq 300$ in females), the trend was maintained but non-significant (table 5). Furthermore, our results were not modified when the date of disease was changed for the date of the first visit with dementia (table 5). Further sensitivity analysis performed to investigate the effect of stroke on the increased risk of dementia with high SUA level (see online supplementary table 4) maintained the association with all-cause dementia but not with vascular or mixed dementia. When participants taking ULT were included in the study sample, results concerning dementia (see online supplementary table 5), brain infarcts, lacunes (see online supplementary table 6) and hippocampal volumes (model 4, $\beta$ (SE) $=-0.012$ $(0.039), \mathrm{p}=0.76)$ were similar to those presented in main analyses. WMHV, however, was slightly associated with high SUA level (models 1-3, see online supplementary table 6).

\section{DISCUSSION}

In our large population-based cohort study, we found increased risk of dementia developing with high SUA level in elderly people, even after multiple adjustments. Analyses by subtype of dementia showed that this association tended to be stronger with vascular or mixed dementia than with $\mathrm{AD}$. This latter finding must be interpreted cautiously however, given the small number of events of vascular dementia.

The association between SUA level and dementia has been debated in the last decade. Historically, low SUA level has been repeatedly reported in neurodegenerative diseases. ${ }^{21}$ The hypothesis often proposed to explain the association is that UA is a natural antioxidant, which might reduce oxidative stress and protect against the detrimental effects of free radicals in the brain. ${ }^{22-25}$ This hypothesis was partly rejected by Hershfield et al, who did not observe any change in oxidative stress markers in patients receiving pegloticase, which greatly lowers SUA level. ${ }^{26}$ Recent in vitro studies found that UA increased oxidative stress and potentiated the neurotoxic effects of amyloid $\beta$ in neuronal cells. ${ }^{27}$ In addition, increasing SUA level with inosine provided no or poor clinical benefit in patients with Parkinson's disease or relapsing-remittent multiple sclerosis. ${ }^{28} 29$

Chen et al, in a meta-analysis of case-control studies $(n=2708$ participants), found no significant difference between patients with $\mathrm{AD}$ and healthy controls in SUA level. ${ }^{10}$ Another recent meta-analysis found lower SUA level in AD but not vascular nor mixed dementia. ${ }^{6}$ Both meta-analyses were however based on cross-sectional studies, with the important limitation that 
Clinical and epidemiological research

Table 4 Association of baseline SUA level and incident dementia: sensitivity analysis in participants not taking NSAIDs ( $\mathrm{n}=1348)$

\begin{tabular}{|c|c|c|c|c|c|c|c|c|c|}
\hline \multirow[b]{2}{*}{ SUA level ( $\mu \mathrm{mol} / \mathrm{L})$} & \multirow{2}{*}{$\frac{\%}{\text { ( } \mathrm{N} \text { event) }}$} & \multicolumn{2}{|l|}{ Model 1* } & \multicolumn{2}{|l|}{ Model $2 \dagger$} & \multicolumn{2}{|l|}{ Model 3‡ } & \multicolumn{2}{|l|}{ Model $4 \S$} \\
\hline & & HR $(95 \% \mathrm{Cl})^{\dagger}$ & $p$ & HR $(95 \% \mathrm{Cl})^{\dagger}$ & $\mathrm{p}$ & HR $(95 \% \mathrm{Cl})^{\dagger}$ & $\mathrm{p}$ & $\mathrm{HR}(95 \% \mathrm{Cl})^{\dagger}$ & $\mathrm{p}$ \\
\hline \multicolumn{10}{|c|}{ All-cause dementia $(n=93)$} \\
\hline$\geq 75$ th percentile & $10.5(36)$ & $1.68(1.10 ; 2.57)$ & 0.016 & $1.89(1.18 ; 3.02)$ & 0.008 & $1.83(1.14 ; 2.93)$ & 0.013 & $1.93(1.20 ; 3.12)$ & 0.007 \\
\hline Q1 & $4.6(16)$ & 1.00 (ref.) & & 1.00 (ref.) & & 1.00 (ref.) & & 1.00 (ref.) & \\
\hline Q2 & $5.8(20)$ & $1.09(0.56 ; 2.11)$ & 0.80 & $1.16(0.59 ; 2.28)$ & 0.66 & $1.19(0.61 ; 2.34)$ & 0.61 & $1.31(0.66 ; 2.58)$ & 0.44 \\
\hline$p$ for linear trend & & & 0.011 & & 0.004 & & 0.005 & & 0.002 \\
\hline \multicolumn{10}{|c|}{ Alzheimer's disease $(n=62)$} \\
\hline$<75$ th percentile & $4.0(39)$ & 1.00 (ref.) & & 1.00 (ref.) & & 1.00 (ref.) & & 1.00 (ref.) & \\
\hline$\geq 75$ th percentile & $6.9(23)$ & $1.56(0.92 ; 2.64)$ & 0.10 & $1.83(1.02 ; 3.27)$ & 0.041 & $1.77(0.99 ; 3.19)$ & 0.06 & $1.87(1.03 ; 3.37)$ & 0.038 \\
\hline Q1 & $3.5(12)$ & 1.00 (ref.) & & 1.00 (ref.) & & 1.00 (ref.) & & 1.00 (ref.) & \\
\hline \multicolumn{10}{|c|}{ Vascular or mixed dementia $(n=18)$} \\
\hline$<75$ th percentile & $0.9(9)$ & 1.00 (ref.) & & 1.00 (ref.) & & 1.00 (ref.) & & 1.00 (ref.) & \\
\hline$\geq 75$ th percentile & $2.8(9)$ & $2.51(0.99 ; 6.33)$ & 0.05 & $2.02(0.65 ; 6.26)$ & 0.22 & $1.82(0.57 ; 5.78)$ & 0.31 & $1.72(0.55 ; 5.41)$ & 0.35 \\
\hline Q1 & $0.6(2)$ & 1.00 (ref.) & & 1.00 (ref.) & & 1.00 (ref.) & & 1.00 (ref.) & \\
\hline Q2 & $1.5(5)$ & $1.87(0.36 ; 9.70)$ & 0.46 & $2.29(0.42 ; 12.42)$ & 0.34 & $2.57(0.47 ; 14.18)$ & 0.28 & $2.35(0.43 ; 12.95)$ & 0.33 \\
\hline Q3 & $0.7(2)$ & $1.00(0.14 ; 7.10)$ & 0.99 & $1.25(0.16 ; 9.45)$ & 0.83 & $1.32(0.17 ; 10.10)$ & 0.79 & $1.25(0.16 ; 9.70)$ & 0.83 \\
\hline Q4 & $2.8(9)$ & $3.38(0.73 ; 15.73)$ & 0.12 & $3.22(0.56 ; 18.62)$ & 0.19 & $3.07(0.52 ; 18.10)$ & 0.22 & $2.73(0.47 ; 16.04)$ & 0.27 \\
\hline$p$ for linear trend & & & 0.08 & & 0.23 & & 0.29 & & 0.34 \\
\hline
\end{tabular}

${ }^{*}$ Model 1: adjusted for education level, gender.

†Model 2: model 1 + BMI. tobacco and alcohol consumption, cholesterol (LDL and HDL), triglycerides, diabetes mellitus, hypertension, interaction between hypertension and time (age at last follow-up or dementia occurrence), history of cardiovascular disease, glomerular filtration rate and APOE-ع4.

¥Model 3: model 2 + aspirin or diuretics.

§Model 4: model $3+$ CRP, IL-6 levels and interaction between IL-6 and time (age at last follow-up or dementia occurrence).

Q1: quartile 1 (lowest quartile); Q1: <260 in men, <209 for women; Q2: 260-299 for men, 209-247 for women; Q3: 299-345 for men, 247-292 for women; Q4: $\geq 345$ for men, $\geq 292$ for women.

APOE, apolipoprotein E; BMI, body mass index; CRP, C reactive protein; HDL, high-density lipoprotein; IL, interleukin; LDL, low-density lipoprotein; NSAIDs, non-steroidal antiinflammatory drugs; SUA, serum uric acid.

physiological and metabolic changes may occur with the onset of AD and may also affect SUA levels. ${ }^{30}$

The sole longitudinal study that assessed the impact of SUA level on incident dementia was conducted in the Rotterdam cohort. Risk of incident dementia was reduced, and cognitive performance in later life was better with high SUA level. ${ }^{31}$ Considering that the mean age at dementia diagnosis is around 85 years $^{32}$ and that the pathological processes leading to dementia can start many years before clinical diagnosis, ${ }^{33}$ the effect of an exposure might depend on when it is measured or on the length of follow-up. Hence, some of the discrepancies between our results and those of the Rotterdam study might be related to differences in the age at SUA measurement $(72.4 \pm 4.1 \mathrm{vs}$ $69.4 \pm 8.4$ years, respectively) or in the duration of follow-up for incident dementia $(8.4 \pm 3.1$ vs $9.0 \pm 3.5$ years, respectively). Moreover, both the Rotterdam and the $3 \mathrm{C}$ study are limited by the availability of a single SUA measurement, and we cannot exclude that potential time-varying effects of SUA could also partly account for the discrepancies observed. Interestingly, in a subsequent cross-sectional study of the same Rotterdam cohort, high SUA level was found associated with white matter atrophy on MRI and worse cognitive performance. ${ }^{34}$

Our findings support several previous studies showing that even mildly elevated SUA levels might contribute to functional brain changes and cognitive decline, especially in older adults. ${ }^{35-38}$ In patients with chronic kidney disease, SUA levels were also inversely associated with MMSE score. ${ }^{39}$ One hypothesis is that the association between higher SUA levels and dementia or lower cognitive performance may be mediated by underlying cerebrovascular disease. ${ }^{13}$ In the present study, we found a non-significant trend towards an association between high SUA level and extensive WMHV, a biomarker of cerebral small vessel disease. ${ }^{15}$ This association was even significant in the larger sample without exclusion of participants taking ULT. Participants receiving ULT are likely patients with gout (ie, with the highest SUA levels), and may thus be particularly exposed to cerebrovascular disease: consistently, we found a higher prevalence of cardiovascular comorbidities in participants taking ULT than in the main sample. This, in addition to the reduction in sample size, may explain why the association between SUA and WMHV was not significant after exclusion of participants taking ULT. Previous studies have shown an association between elevated SUA level and WMH or white matter atrophy. ${ }^{34} 40-42$ Brain infarcts and lacunes were not associated with SUA levels in our study. However, the association between SUA levels and vascular or mixed dementia, but not all dementia, disappeared after adjustment on interim strokes, suggesting a mediating effect of stroke for the vascular or mixed dementia subtype only.

Inflammation may be another potential mediator of the association between high SUA levels and vascular dementia risk. In 


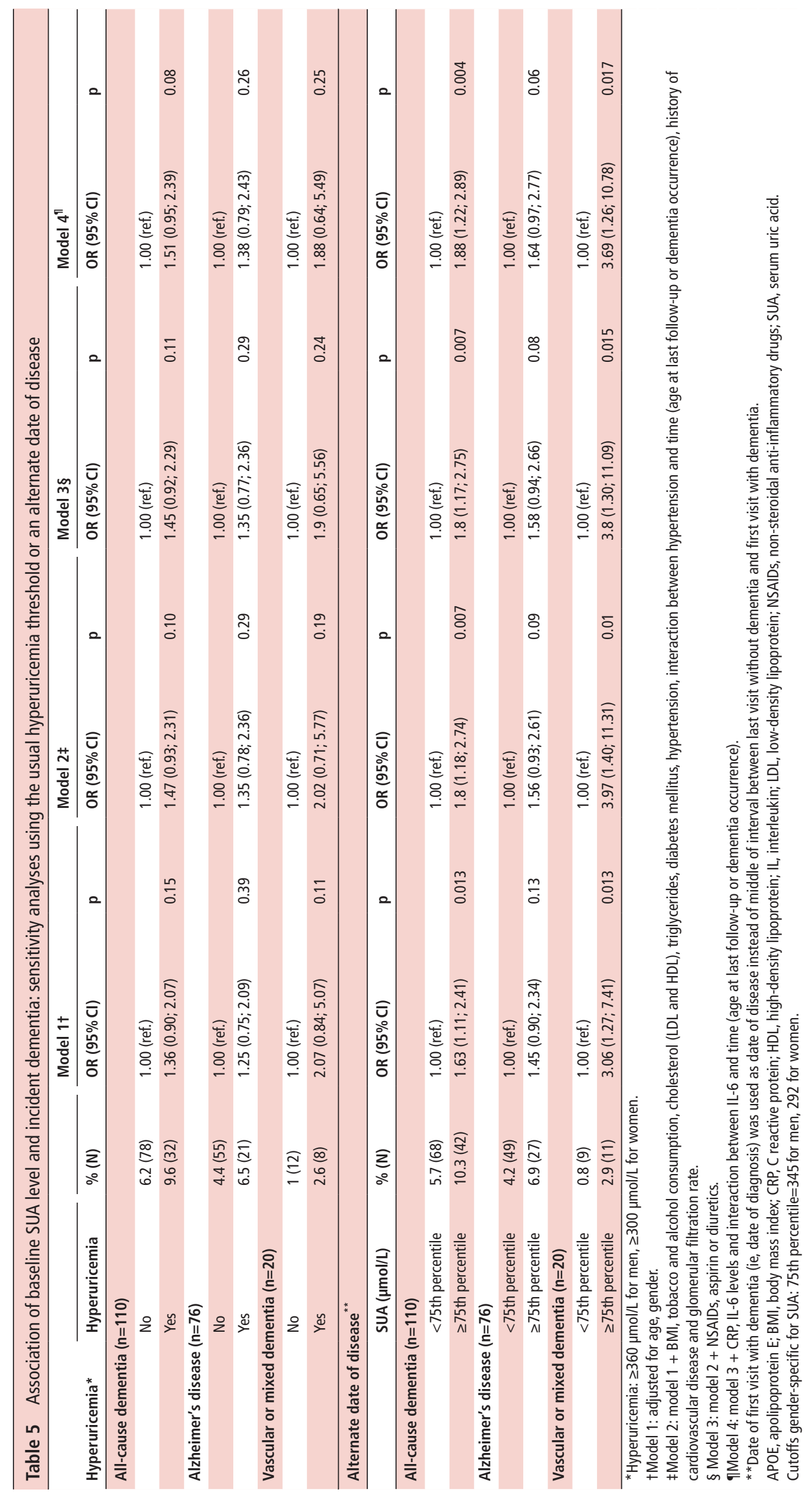


line with this hypothesis, individuals with high SUA levels were more likely to have high CRP and IL-6 levels at baseline in the present study. Some data support that systemic inflammation might mediate the effects of UA on vascular damage, including WMH. $^{43} 44$

Because NSAIDs might impact the risk of dementia by modulating oxidative stress or systemic inflammation, we also explored their effect in sensitivity analyses after excluding participants taking NSAIDs. While the overall risk of dementia was not affected, the association with $\mathrm{AD}$ was strengthenedconsistently with previous evidence showing a decreased risk of $\mathrm{AD}$ in NSAIDs users. ${ }^{45}$ Conversely, the association with vascular or mixed dementia lost its significance. This latter finding must be interpreted with caution given the small number of events.

While our main analysis was conducted after exclusion of participants taking ULT, the association between SUA and dementia was substantially unchanged if including participants taking ULT, supporting the robustness of our findings. The subgroup of participants under ULT was too small to conduct detailed analyses on the association of SUA with dementia in this subgroup. The effect of ULT on the risk of dementia remains to be elucidated in further studies.

Our study has several strengths. It is a large prospective cohort study with a follow-up of more than 12 years. Diagnosis of dementia was based on a thorough three-step procedure, including the review of each diagnosis by an independent committee of experts, and different subtypes of dementia were evaluated. Each participant was thoroughly screened for a large number of factors associated with cognitive impairment or SUA level, and brain MRI markers were assessed in parallel. Importantly, gender-dependent cutoffs of SUA were determined in order to avoid any gender bias in our analyses, and various sensitivity analyses were realised. Several limitations should, however, be considered. First, our participants are not perfectly representative of the French general population of the same age range since they are volunteering for extensive investigations and follow-up. This is a common limitation of prospective population-based studies, regardless of the sampling method used. However, as suggested by the Paquid study, population-based samples may become more representative of the general population after a long follow-up period. ${ }^{46}$ This may limit the generalisability of our findings. It is important to note that our goal was to evaluate the association of dementia with SUA levels and not with hyperuricemia or gout per se. Hence, inclusion criteria were not based on SUA levels, and therefore results displayed herein are not generalisable to hyperuricemia or gout cohorts. Second, the old age of our participants implies that we are lacking SUA measurements in midlife, which may better reflect life-long exposure than late-life measurements. Moreover, the latter may be modified by behavioural changes, initiation of treatments and concomitant age-related chronic diseases. Third, survival bias cannot be ruled out, as persons with high SUA levels may have died from cardiovascular disease before the lower age limit of 65 years for enrolment in the 3C-Dijon study. However, given the association of high SUA with increased risk of cardiovascular events this would be expected to attenuate our findings. ${ }^{47}$ Conversely, we cannot exclude that AD might have occurred prematurely in some people with low SUA levels before 65 years, who would not have been selected in the present study since dementia was an exclusion criterion. This selection bias due to the depletion of susceptibles could explain the null findings behind SUA and $\mathrm{AD}$ in this study. Fourth, the availability of only a single measurement of SUA in our study prevents us from investigating potential time-varying effects of SUA.

In conclusion, in a large prospective cohort of elderly people, high SUA levels were associated with increased risk of dementia, especially vascular or mixed dementia. These results require confirmation in other prospective large-scale studies including younger individuals with long follow-up duration and ideally repeated measurements of SUA.

Acknowledgements The authors acknowledge the contribution of the Three-City Dijon MRI study participants.

Contributors All authors contributed substantially to the conception or design of the work, or the acquisition, analysis or interpretation of data for the work; drafted the work or revised it critically for important intellectual content; gave their final approval of the version to be published; and agreed to be accountable for all aspects of the work in ensuring that questions related to the accuracy or integrity of any part of the work are appropriately investigated and resolved.

Funding The 3-City Study is conducted under a partnership agreement among the Institut National de la Santé et de la Recherche Médicale (INSERM), the Victor Segalen-Bordeaux II University, and Sanofi-Aventis. The Fondation pour la Recherche Médicale funded the preparation and initiation of the study. The 3C Study is also supported by the Caisse Nationale Maladie des Travailleurs Salariés, Direction Générale de la Santé, Mutuelle Générale de I'Education Nationale (MGEN), Institut de la Longévité, Conseils Régionaux of Aquitaine and Bourgogne, Fondation de France, Caisse Nationale de Solidarité et Longévité (CNSA) and Ministry of ResearchINSERM Programme "Cohortes et collections de données biologiques." The funders had no role in study design, data collection and analysis, decision to publish, or preparation of the manuscript.

\section{Competing interests None declared.}

Ethics approval The Ethics Committee of Kremlin-BicÃ $f \hat{A}^{a}$ tre University Hospital approved study protocols, and each participant signed a written informed consent.

Provenance and peer review Not commissioned; externally peer reviewed.

Data sharing statement Data available upon request to the $3 C$ data access committee. Please email requests to: E3C.CoordinatingCenter@gmail.com and E3C. U708@inserm.fr.

(C) Article author(s) (or their employer(s) unless otherwise stated in the text of the article) 2018. All rights reserved. No commercial use is permitted unless otherwise expressly granted.

\section{REFERENCES}

1 Feig DI, Kang DH, Johnson RJ. Uric acid and cardiovascular risk. N Engl J Med 2008;359:1811-21

2 Zhu Y, Pandya BJ, Choi HK. Comorbidities of gout and hyperuricemia in the US general population: nhanes 2007-2008. Am J Med 2012;125:679-87.

3 Richette P, Perez-Ruiz F, Doherty M, et al. Improving cardiovascular and renal outcomes in gout: what should we target? Nat Rev Rheumatol 2014;10:654-61.

4 Khanna D, Fitzgerald JD, Khanna PP, et al. 2012 American College of Rheumatology guidelines for management of gout. Part 1: systematic nonpharmacologic and pharmacologic therapeutic approaches to hyperuricemia. Arthritis Care Res 2012;64:1431-46

5 Richette P, Doherty M, Pascual E, et al. 2016 updated EULAR evidence-based recommendations for the management of gout. Ann Rheum Dis 2017;76:29-42.

6 Khan AA, Quinn TJ, Hewitt J, et al. Serum uric acid level and association with cognitive impairment and dementia: systematic review and meta-analysis. Age 2016:38:16.

7 Maxwell SR, Thomason H, Sandler D, et al. Antioxidant status in patients with uncomplicated insulin-dependent and non-insulin-dependent diabetes mellitus. Eur J Clin Invest 1997;27:484-90

8 Shen L, Ji HF, Hf J. Low uric acid levels in patients with Parkinson's disease: evidence from meta-analysis. BMJ Open 2013;3:e003620.

9 Abraham A, Drory VE. Influence of serum uric acid levels on prognosis and survival in amyotrophic lateral sclerosis: a meta-analysis. J Neurol 2014;261:1133-8.

10 Chen X, Guo X, Huang R, et al. Serum uric acid levels in patients with Alzheimer's disease: a meta-analysis. PLoS One 2014;9:e94084.

11 Hong JY, Lan TY, Tang GJ, et al. Gout and the risk of dementia: a nationwide population-based cohort study. Arthritis Res Ther 2015:17:139.

12 Lu N, Dubreuil M, Zhang Y, et al. Gout and the risk of Alzheimer's disease: a population-based, BMl-matched cohort study. Ann Rheum Dis 2016;75:547-51.

13 Vannorsdall TD, Jinnah HA, Gordon B, et al. Cerebral ischemia mediates the effect of serum uric acid on cognitive function. Stroke 2008:39:3418-20.

14 Caroli A, Frisoni GB; Alzheimer's Disease Neuroimaging Initiative. The dynamics of Alzheimer's disease biomarkers in the Alzheimer's Disease Neuroimaging Initiative cohort. Neurobiol Aging 2010;31:1263-74. 
15 Debette S, Markus HS. The clinical importance of white matter hyperintensities on brain magnetic resonance imaging: systematic review and meta-analysis. BMJ 2010;341:c3666.

16 Jack CR, Petersen RC, Xu Y, et al. Rates of hippocampal atrophy correlate with change in clinical status in aging and AD. Neurology 2000;55:484-90.

173 C Study Group. Vascular factors and risk of dementia: design of the Three-City Study and baseline characteristics of the study population. Neuroepidemiology 2003;22:316-25.

18 McKhann G, Drachman D, Folstein M, et al. Clinical diagnosis of Alzheimer's disease: report of the NINCDS-ADRDA Work Group under the auspices of Department of Health and Human Services Task Force on Alzheimer's Disease. Neurology 1984;34:939-44.

19 Román GC, Tatemichi TK, Erkinjuntti T, et al. Vascular dementia: diagnostic criteria for research studies. Report of the NINDS-AIREN International Workshop. Neurology 1993;43:250-60.

20 Rho YH, Zhu Y, Choi HK. The epidemiology of uric acid and fructose. Semin Nephrol 2011;31:410-9.

21 Kutzing MK, Firestein BL. Altered uric acid levels and disease states. J Pharmacol Exp Ther 2008;324:1-7.

22 Bowman GL, Shannon J, Frei B, et al. Uric acid as a CNS antioxidant. J Alzheimers Dis 2010;19:1331-6.

23 Cervellati C, Romani A, Seripa D, et al. Oxidative balance, homocysteine, and uric acid levels in older patients with late Onset Alzheimer's Disease or Vascular Dementia. J Neurol Sci 2014;337:156-61.

24 Hatanaka H, Hanyu H, Fukasawa R, et al. Differences in peripheral oxidative stress markers in Alzheimer's disease, vascular dementia and mixed dementia patients. Geriatr Gerontol Int 2015;15 Suppl 1(Suppl 1):53-8.

25 Schrag M, Mueller C, Zabel M, et al. Oxidative stress in blood in Alzheimer's disease and mild cognitive impairment: a meta-analysis. Neurobiol Dis 2013;59:100-10.

26 Hershfield MS, Roberts LJ, Ganson NJ, et al. Treating gout with pegloticase, a PEGylated urate oxidase, provides insight into the importance of uric acid as an antioxidant in vivo. Proc Natl Acad Sci U SA 2010;107:14351-6.

27 Desideri G, Gentile R, Antonosante A, et al. Uric Acid amplifies A $\beta$ Amyloid Effects involved in the Cognitive Dysfunction/Dementia: evidences from an Experimental Model in Vitro. J Cell Physiol 2017;232:1069-78.

28 Gonsette RE, Sindic C, D'hooghe MB, et al. Boosting endogenous neuroprotection in multiple sclerosis: the ASsociation of Inosine and interferon beta in relapsingremitting multiple sclerosis (ASIIMS) trial. Mult Scler 2010;16:455-62.

29 Schwarzschild MA, Ascherio A, Beal MF, et al. Inosine to increase serum and cerebrospinal fluid urate in Parkinson disease: a randomized clinical trial. JAMA Neurol 2014;71:141-50.
30 Lopes da Silva S, Vellas B, Elemans S, et al. Plasma nutrient status of patients with Alzheimer's disease: Systematic review and meta-analysis. Alzheimers Dement 2014;10:485-502.

31 Euser SM, Hofman A, Westendorp RG, et al. Serum uric acid and cognitive function and dementia. Brain 2009;132:377-82.

32 Satizabal CL, Beiser AS, Chouraki V et al. Incidence of dementia over three decades in the Framingham Heart Study. N Engl J Med 2016;374:523-32.

33 Jack CR, Knopman DS, Jagust WJ, et al. Tracking pathophysiological processes in Alzheimer's disease: an updated hypothetical model of dynamic biomarkers. Lancet Neurol 2013;12:207-16.

34 Verhaaren BF, Vernooij MW, Dehghan A, et al. The relation of uric acid to brain atrophy and cognition: the Rotterdam scan Study. Neuroepidemiology 2013:41:29-34.

35 Schretlen $D J$, Inscore $A B$, Jinnah $H A$, et al. Serum uric acid and cognitive function in community-dwelling older adults. Neuropsychology 2007;21:136-40.

36 Cicero AF, Desideri G, Grossi G, et al. Serum uric acid and impaired cognitive function in a cohort of healthy young elderly: data from the Brisighella Study. Intern Emerg Med 2015;10:25-31.

37 Ruggiero C, Cherubini A, Lauretani F, et al. Uric acid and dementia in communitydwelling older persons. Dement Geriatr Cogn Disord 2009;27:382-9.

38 Beydoun MA, Canas JA, Dore GA, et al. Serum uric acid and its Association with Longitudinal Cognitive Change among Urban adults. J Alzheimers Dis 2016;52:1415-30.

39 Afsar B, Elsurer R, Covic A, et al. Relationship between uric acid and subtle cognitive dysfunction in chronic kidney disease. Am J Nephrol 2011;34:49-54.

40 Schretlen DJ, Inscore AB, Vannorsdall TD, et al. Serum uric acid and brain ischemia in normal elderly adults. Neurology 2007;69:1418-23.

41 Fanning JP, Wong AA, Fraser JF. The epidemiology of silent brain infarction: a systematic review of population-based cohorts. BMC Med 2014;12:119.

42 Shih CY, Chen CY, Wen CJ, et al. Relationship between serum uric acid and cerebral white matter lesions in the elderly. Nutr Metab Cardiovasc Dis 2012;22:154-9.

43 Perez-Ruiz F, Becker MA. Inflammation: a possible mechanism for a causative role of hyperuricemia/gout in cardiovascular disease. Curr Med Res Opin 2015;31 Suppl 2:9-14.

44 Satizabal CL, Zhu YC, Mazoyer B, et al. Circulating IL-6 and CRP are associated with MRI findings in the elderly: the 3C-Dijon Study. Neurology 2012;78:720-7.

45 Wang J, Tan L, Wang HF, et al. Anti-inflammatory drugs and risk of Alzheimer's disease: an updated systematic review and meta-analysis. J Alzheimers Dis 2015;44:385-96.

46 Helmer C, Pérès K, Letenneur L, et al. Dementia in subjects aged 75 years or over within the PAQUID cohort: prevalence and burden by severity. Dement Geriatr Cogn Disord 2006;22:87-94.

47 Zhao G, Huang L, Song M, et al. Baseline serum uric acid level as a predictor of cardiovascular disease related mortality and all-cause mortality: a meta-analysis of prospective studies. Atherosclerosis 2013;231:61-8. 